\title{
PROVENIENZFORSCHUNG INS MUSEUM: AUSSTELLUNGSER- ÖFFNUNG UND RESTITUTION DER WIRTSCHAFTSUNIVERSITÄT WIEN IM TECHNISCHEN MUSEUM WIEN
}

\author{
von Johannes Koll und Regina Zodl
}

Zusammenfassung: Am 4. Oktober 2017 wurde die Integrierung der Privatbibliothek des österreichischen Petrochemikers Dr. Leopold Singer (1869-1942), die zwei Jahre vorher von der Wirtschaftsuniversität Wien an dessen Erben und Erbinnen restituiert worden war, in die Dauerausstellung des Technischen Museums Wien gefeiert. Erstmalig werden damit Ergebnisse bibliothekarischer Provenienzforschung in einem international renommierten Museum der Öffentlichkeit zugänglich gemacht. Zugleich wurde ein Buch restituiert, das bis zum „Anschluss" Österreichs Dr. Martin Singer (1874-1950) gehört hatte.

Schlüsselwörter: Wirtschaftsuniversität Wien; Universitätsbibliothek; NS-Provenienzforschung; Restitution; Technisches Museum Wien; Dr. Leopold Singer; Dr. Martin Singer; Gedenkkultur; Ausstellung

\section{PROVENANCE RESEARCH ON DISPLAY: EXHIBITION OPENING AND RESTITUTION BY VIENNA UNIVERSITY OF ECONOMICS AND BUSINESS AT VIENNA TECHNICAL MUSEUM}

Abstract: On October 4, 2017, the Vienna Technical Museum celebrated the integration of books from the private library of petrochemical specialist Dr. Leopold Singer (1869-1942) into the museum's permanent collection. The works had been restored to the rightful heirs by the University Library of the WU (Wirtschaftsuniversität Wien, Vienna University of Economics and Business) two years previously. With this exhibition, the results of a library's provenance research are on display to the public for the first time in a prominent museum. During the ceremony, a further book was restituted that had belonged to Dr. Martin Singer (1874-1950) before Austria's annexation to Nazi Germany.

Keywords: Vienna University of Economics and Business; university library; NS provenance research; restitution; Vienna Technical Museum; Dr. Leopold Singer; Dr. Martin Singer; culture of remembrance; exhibition 


\section{Inhalt}

1. Museale Präsentation universitärer Provenienzforschung

2. Familie Singer und die österreichische Erdölgeschichte

3. Provenienzforschung und Restitutionen der WU

4. Aufarbeitung der Vergangenheit an der WU

\section{Museale Präsentation universitärer Provenienzforschung}

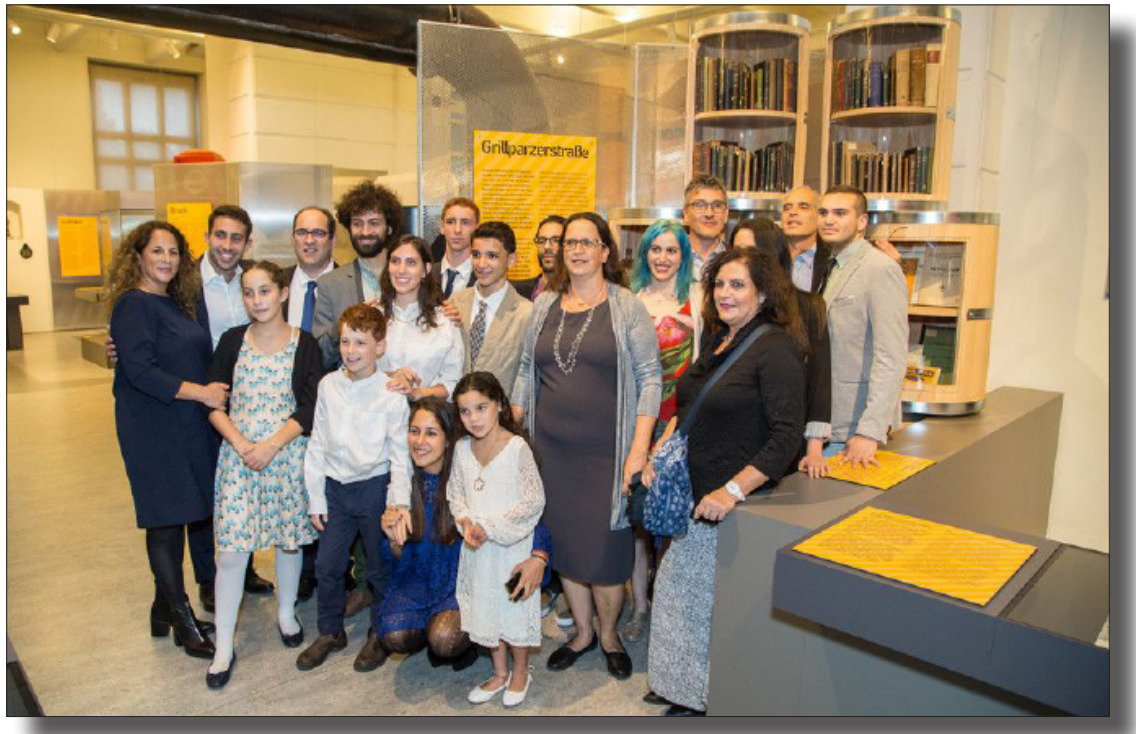

Abb. 1: Die Nachfahren von Leopold und Martin Singer bei der Feier im Technischen Museum Wien am 4. Oktober 2017 (Copyright: TMW/APA-Fotoservice/Krisztian Juhasz)

Am 4. Oktober 2017 fand im Technischen Museum Wien (TMW) eine Feier statt, in die die Provenienzforschung an der Wirtschaftsuniversität Wien (WU) in zweifacher Hinsicht eingebunden war. Zum einen konnte ein Buch an die Erben und Erbinnen des jüdischen Chemikers Dr. Martin Singer (geb. 1874 in Wien, gest. 1950 in Sydney) restituiert werden. Zum anderen wurde die Neugestaltung der TMW-Abteilung zu Erdöl und Erdgas eröffnet, in die ein Großteil der Buchbestände von Dr. Leopold Singer (geb. 1869 in Wien, gest. 1942 in London), dem älteren Bruder von Martin Singer, inkorporiert wurde. Der Bezug dieser Bücher zur WU liegt darin, dass sie 1942 auf dem Wege der „Arisierung“ in den Besitz der Hochschule für Welthandel, der Vorgängerin der WU, gekommen waren. 
Die Eingliederung der Bibliothek von Leopold Singer in einen Teil der Dauerausstellung des TMW verlieh der Eröffnungsfeier vom 4. Oktober 2017 eine besondere Bedeutung: Erstmalig im deutschsprachigen Raum sind von nun an Ergebnisse bibliothekarischer Provenienzforschung in einem Museum der Öffentlichkeit zugänglich. Dadurch wiederum eröffnen WU und TMW nachfolgenden Generationen breitenwirksam die Möglichkeit, mit dem staatlich organisierten Raub von Kulturgut einen spezifischen Aspekt der Verfolgung von Juden und Jüdinnen durch das NS-Regime vor Augen zu führen.

\section{Familie Singer und die österreichische Erdölgeschichte}

Darüber hinaus erleichtert die Aufnahme der Bibliothek von Leopold Singer in einen Teil der Dauerausstellung des TMW eine Neubewertung der Bedeutung der Familie Singer für die österreichische Erdölgeschichte. ${ }^{1}$ Der Vater von Martin und Leopold, Wilhelm Singer (1843-1934), war der erste Unternehmer, der Erdöl aus dem Russischen Reich per Schiff nach London lieferte. Um 1890 besaß er eine Raffınerie im heutigen Rumänien und ließ in Triest ein Tanklager errichten. Mit Martin und Leopold Singer traten mindestens zwei von sechs Kindern des Ehepaares Wilhelm und Rosalie Singer (geborene Klein) in die väterlichen Fußstapfen.

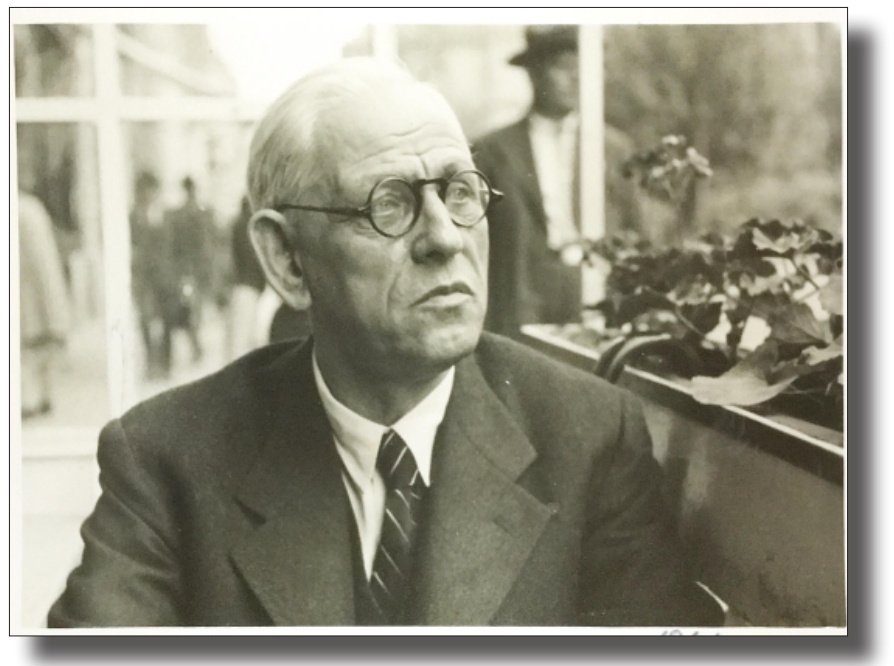

Abb. 2: Martin Singer 1944 in Sydney, fotografiert von seinem Sohn Kurt Singer (Copyright: Robert Lehrer) 
Martin Singer war im Anschluss an die Matura, die er 1892 an einem Wiener Gymnasium ablegte, zwei Jahre lang am Eidgenössischen Polytechnikum (heute: ETH Zürich) eingeschrieben und wechselte 1894 zur Fortsetzung des Studiums für weitere zwei Jahre an die Universität Würzburg. Hier wurde er 1897 mit der Dissertation „Über Diazosulfone und Ketone“ promoviert. Bis zum „Anschluss" Österreichs war er sodann in Führungspositionen von mehreren Unternehmen in Österreich, Ungarn und Rumänien tätig. Mit seiner früh verstorbenen Ehefrau Bella Scheinberger (1881-1905) hatte er zwei Kinder: Kurt (1900-1948) und Edith (1902-1991). Nur wenige Tage nach dem Einmarsch der deutschen Wehrmacht in Österreich meldete er sich von seiner Wohnung in der Wipplingerstraße 32 (1. Wiener Gemeindebezirk) ab. Er emigrierte nach Australien und ließ sich in Sydney nieder. Hier starb er am 9. Januar 1950.

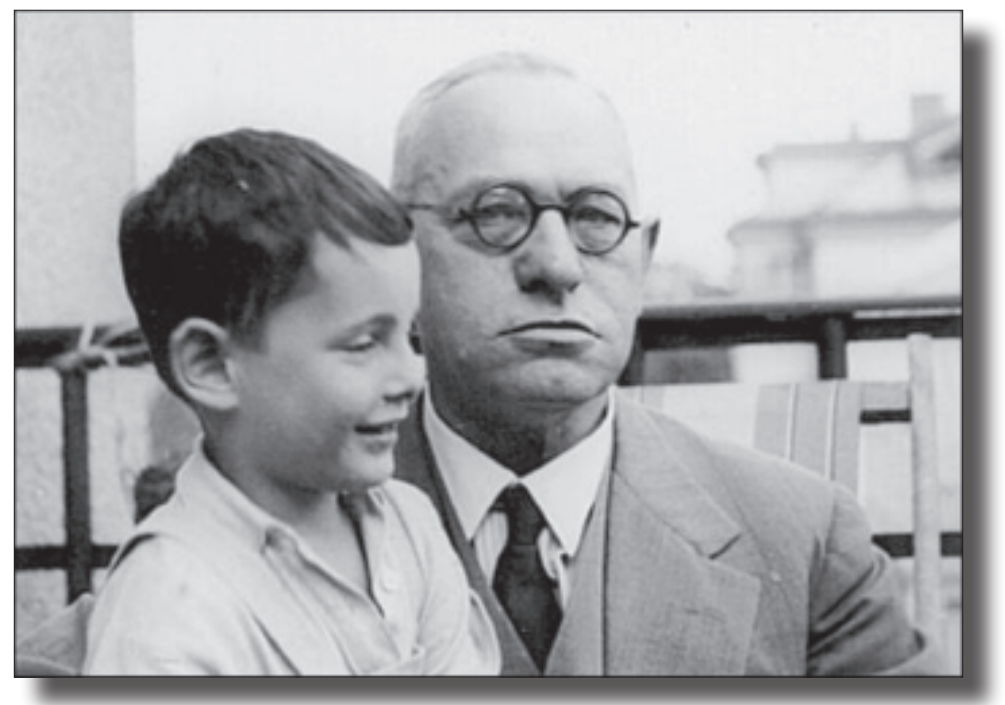

Abb. 3: Leopold Singer mit Enkel Thomas Singer, vermutlich 1934/35 in Wien (Copyright: Amir Singer)

Leopold Singer, der zweitälteste Sohn von Wilhelm und Rosalie, begann 1887 das Studium der Technischen Chemie an der k.k. Technischen Hochschule in Wien. Wie später sein jüngerer Bruder Martin schrieb sich Leopold am Eidgenössischen Polytechnikum in Zürich ein, und zwar im Jahr 1889. Seine Promotion bestritt er vier Jahre später an der Universität Zürich mit einer Dissertation über das Thema „Beiträge zur Theorie der Petroleumbildung“. Leopold Singer arbeitete in der väterlichen Raf- 
finerie in Orschowa/Orşova (heute in Rumänien), erwarb eine Reihe von Patenten (zum Teil zusammen mit seinem Bruder Martin) und war seit 1909 ständiger Mitarbeiter der Fachzeitschrift „Petroleum“. Außerdem wirkte er an mehreren Bänden des Standardwerks „Das Erdöl“ mit, das Hans Höfer von Heimhalt ab 1909 zusammen mit Carl Engler herausgab, und publizierte in Fachzeitschriften. 1903 wurde Singer Direktor der Fanto-Werke in Pardubitz/Pardubice (heute: Tschechische Republik). Von 1914 bis 1918 war er Geschäftsführer der Mineralölwerke Rhenania AG in Monheim. Seit 1918 war Leopold Singer auf nationaler und internationaler Ebene als Berater für die Erdölindustrie tätig, etwa als Konsulent der Allgemeinen Depositenbank in Wien sowie ab 1926 als Chemischer Konsulent der „Universal Oil Company of Chicago“. Außerdem beriet er das im Mai 1918 eröffnete Technische Museum Wien in seiner Gründungsphase im Hinblick auf die Schausammlung zum Themenbereich Petrochemie. Zwischen 1919 und 1938 lebte der renommierte Erdölfachmann mit seiner Familie in einer Wohnung in der Grillparzerstraße 7 (1. Wiener Gemeindebezirk), zwischen Universität und Rathaus gelegen. Am 25. März 1939 meldete sich Singer nach Rumänien ab. Später emigrierte er nach Großbritannien. Am 10. Juni 1942 verstarb Leopold Singer in London.

Seine Büchersammlung konnte er nicht ins Exil mitnehmen. Sie wurde zu einem nicht eruierbaren Zeitpunkt von der Gestapo beschlagnahmt. Mutmaßlich 1942 bot die „Verwertungsstelle für jüdisches Umzugsgut der Gestapo" (VUGESTA) den Bestand, der Bücher in mehreren Sprachen enthielt, zum Kauf an. Die Hochschule für Welthandel, die sich nicht zuletzt seit April 1940 im Rahmen der Kurse der „Südost-Stiftung des Mitteleuropäischen Wirtschaftstages Berlin zur Heranbildung junger Kaufleute für Südost-Europa“ mit Rohstofftechnik und -wirtschaft befasste, ${ }^{2}$ erwarb schließlich die Bibliothek.

\section{Provenienzforschung und Restitutionen der WU}

Sowohl die Ausstellung zu Leopold Singer als auch die Restitution nach Martin Singer sind aus dem Provenienzforschungsprojekt hervorgegangen, das seit 2010 an der Universitätsbibliothek der WU durchgeführt wird. In diesem Rahmen konnten 2013 zwei sowie im folgenden Jahr weitere zehn Bücher restituiert werden.

Die umfangreichste Restitution fand am 1. Oktober 2015 statt: Hierbei wurden 696 Bücher an die Erben und Erbinnen nach Leopold Singer resti- 
tuiert. ${ }^{3}$ Entsprechend dem Wunsch von dessen Nachfahren, den größten Teil dieses Bestandes öffentlich zugänglich zu machen, haben Regina Zodl und Doz. Dr. Johannes Koll (WU) in den folgenden Monaten zusammen mit Dr. Christian Klösch (TMW) und mit freundlicher Unterstützung durch Albena Zlatanova (Nationalfonds der Republik Österreich für Opfer des Nationalsozialismus) die Einbindung dieser Büchersammlung in die Dauerausstellung des TMW vorbereitet. Mit der Eröffnung der neugestalteten Abteilung zu Erdöl und Erdgas können somit Ergebnisse von Provenienzforschung sichtbar gemacht und zugleich die Erinnerung an einen international anerkannten österreichischen Erdölfachmann wachgehalten bzw. neu angeregt werden. Dass seinerzeit die Expertise von Leopold Singer im neugegründeten TMW gefragt war, verleiht der Neugestaltung rückblickend eine weitere besondere Note.

Zusammen mit der Ausstellungseröffnung vom 4. Oktober 2017 konnte die vorläufig letzte Restitution verbunden werden, eben jene nach Martin Singer. Grußworte und Ansprachen hielten auf dem Festakt Dr. Gabriele Zuna-Kratky (Generaldirektorin des TMW), Mag. Hannah Lessing (Nationalfonds) sowie Vizerektor Univ.-Prof. Dr. Stefan Pichler und Bibliotheksdirektor Nikolaus Berger (WU).

\section{Aufarbeitung der Vergangenheit an der WU}

Das Provenienzforschungsprojekt fügt sich in eine Reihe von Forschungsinitiativen der WU ein, die sich kritisch mit der eigenen Vergangenheit auseinandersetzen. So wurde im Rahmen des Gedenkprojekts ein Internet-Gedenkbuch erarbeitet, in dem Kurzbiographien zu Studierenden, Doktoranden und Doktorandinnen, Lehrenden und Verwaltungsangestellten der Hochschule für Welthandel zusammengestellt sind, die seit dem „Anschluss“ Österreichs aus politischen und/oder rassischen Gründen vom NS-Regime verfolgt wurden. ${ }^{4}$ Die Namen dieser Personen bilden auch das Mahnmal, das Alexander Felch, Absolvent der Akademie der bildenden Künste Wien, auf dem WU-Campus am Rande des Praters geschaffen hat. ${ }^{5}$ Außerdem ist aus dem Gedenkprojekt ein wissenschaftlicher Sammelband mit Beiträgen zu „Säuberungen“ an österreichischen Hochschulen zwischen 1934 und 1945 hervorgegangen. ${ }^{6}$ Schließlich hat sich die WU am 9. Juni 2015 im Rahmen einer prominent besetzten Podiumsveranstaltung mit der „Affäre Borodajkewycz“ auseinandergesetzt, die die NS-Zeit auf problematische Weise mit der Geschichte der Hochschule für Welthandel in der Nachkriegszeit verbindet. ${ }^{7}$ 


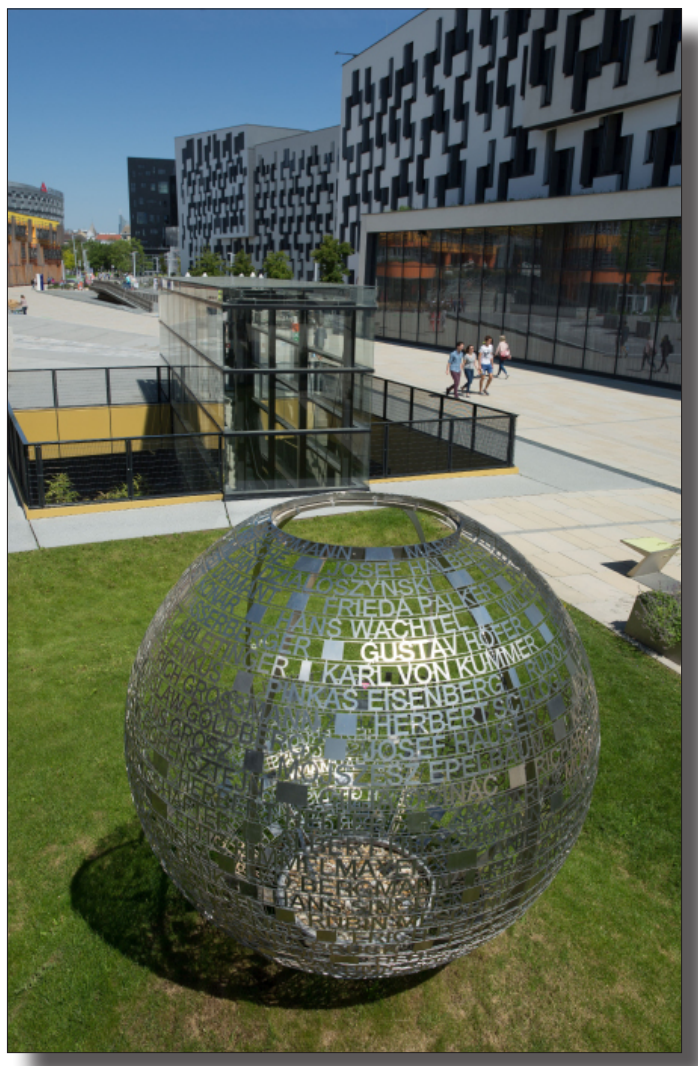

Abb. 4: Das Mahnmal auf dem WU-Campus (Copyright: Jürgen Angel)

Doz. Dr. Johannes Koll Wirtschaftsuniversität Wien, Universitätsarchiv E-Mail: johannes.koll@wu.ac.at

Regina Zodl Wirtschaftsuniversität Wien, Universitätsarchiv E-Mail: regina.zodl@wu.ac.at 
1 Die Quellenbelege zu den folgenden Ausführungen befinden sich in den Projektunterlagen der WU. Für die Informationen zu Wilhelm Singer danken wir Dr. Christian Klösch (TMW).

2 Siehe hierzu: Roumiana Preshlenova, Elitenbildung. Die „Südoststiftung des Mitteleuropäischen Wirtschaftstags Berlin an der Hochschule für Welthandel in Wien“, in: Carola Sachse (Hrsg.), „Mitteleuropa“ und „Südosteuropa“ als Planungsraum. Wirtschafts- und kulturpolitische Expertisen im Zeitalter der Weltkriege (= Diktaturen und ihre Überwindung im 20. und 21. Jahrhundert, Bd. 4), Göttingen 2010, S. 391-417.

3 Siehe den Beitrag von Roswitha Hammer und Regina Zodl unter http:// blog.wu.ac.at/2015/10/restitution-von-buechern-an-die-erben-underbinnen-nach-dr-leopold-singer/ (Zugriff: 30.10.2017).

4 Gedenkbuch für die Opfer des Nationalsozialismus an der Hochschule für Welthandel 1938-1945, http://gedenkbuch.wu.ac.at/.

5 Ein Videomitschnitt der Eröffnung des Mahnmals am 8. Mai 2014 ist abrufbar unter https://www.wu.ac.at/wutv/show/clip/20140508mahnmal/ (Zugriff: 30.10.2017).

6 Johannes Koll (Hrsg.), „Säuberungen“ an österreichischen Hochschulen 1934-1945. Voraussetzungen, Prozesse, Folgen, Wien/Köln/Weimar 2017.

7 Zu dieser Veranstaltung siehe den Bericht im Nachrichtenmagazin ZIB 2 des ORF vom 9. Juni 2015 (ORF 2, 22.00 Uhr); Videomitschnitt unter https://www.wu.ac.at/wutv/show/clip/20150609-borodajkewycz-complete/ sowie Johannes Koll, Schatten brauner Vergangenheit - die „Affäre Borodajkewycz“, in: WU-Blog 6/2015, http://blog.wu.ac.at/2015/06/ schatten-brauner-vergangenheit-die-affaere-borodajkewycz) (Zugriffe: 30.10.2017). Zur „Affäre Borodajkewycz“ siehe Rafael Kropiunigg, Eine österreichische Affäre: der Fall Borodajkewycz, Wien 2015.

Dieses Werk ist lizenziert unter einer

Creative-Commons-Lizenz Namensnennung 4.0 International 